\title{
Metastatic Adenocarcinoma of the Lung Mimicking Spinal Tuberculosis
}

\author{
Zamzuri Z a, Adham SY a, Shukrimi A ${ }^{\mathrm{a}}$, Azril MA a , Amran R \\ aDepartment of Orthopaedics, Traumatology \& Rehabilitation, Kulliyyah of Medicine, \\ International Islamic University Malaysia, Kuantan, Pahang; Malaysia \\ bepartment of Radiology, Kulliyyah of Medicine. International Islamic University Malaysia, \\ Kuantan, Pahang; Malaysia
}

\begin{abstract}
Clinically, it is sometimes difficult to distinguish an infection of the spine from a metastasis. Spinal tuberculosis is common according to its endemic region and adenocarcinoma of the lung is also rising in the incidence worldwide. Similar presentations, with unknown primary, clinical findings and hematological investigations rarely conclude a true diagnosis. Radiologically, the hallmark of spinal infection is erosion of adjacent vertebral endplates and narrowing of the disc space with or without a paravertebral shadow. Metastasis typically does not involve the disc space with erosion of the adjacent vertebral endplates. It usually presents as a lytic/sclerotic lesion in the vertebral body or "winkle owl" sign. These distinguishing features of infection versus metastasis are not certainties. Biopsy is mandatory whenever in doubt or patient is not responded with provisional treatment. The author presents a case with so-called radiological features of spinal tuberculosis infection, which turns out to be a metastatic adenocarcinoma of the lung.
\end{abstract}

KEYWORDS: Tuberculosis, lung cancer, adenocarcinoma, metastasis

\section{INTRODUCTION}

In many parts of the world, adenocarcinoma of the lung shows an increase in its incidence. It is the second most common histological type of lung carcinoma after squamous-cell carcinoma in many series. Adenocarcinoma naturally grows slowly and usually metastasize later in it course than small cell lung carcinoma and consequently, surgery offers the best chance of cure when the tumor is resectable at diagnosis.

The spine, pelvis, femur, ribs and skull are the most frequently involved region for bone metastasis. Systemic manifestations of lung cancer are present in more than $50 \%$ of cases at diagnosis and $10-15 \%$ of lung cancers are detected incidentally in the asymptomatic patients via chest radiographs. As we are in an endemic area for tuberculosis, it is common that anti tuberculous drug is prescribed for the suspected cases of Potts disease based on the clinical presentation and MRI without histology confirmation.

Corresponding author:

Dr Zamzuri bin Zakaria

Associate Professor,

Department Of Orthopaedics, Traumatology \&

Rehabilitation,

Kulliyyah of Medicine,

International Islamic University Malaysia,

Jalan Hospital, 25150 Kuantan, Pahang, Malaysia

Email: zamdr@yahoo.co.uk
However, the distinction in imaging studies between tuberculosis and metastasis of the spine can be very difficult. Throughout the years several reports have addressed this topic. Data on the frequency of this dilemma could not be found. Many reports from non endemic tuberculosis areas have addressed the importance of spinal tuberculosis in the differential diagnoses of a tumour, as mimicking presentation sometimes causing unnecessary radiotherapy treatment in the spinal tuberculosis patient. Here, we report a rare case of metastatic adenocarcinoma of the lung to the spine which initially thought as spinal tuberculosis.

\section{CASE REPORT}

A 56-year-old Malay man presented with low back pain and bilateral lower limbs weakness for three months. The pain was pricking in nature, localized at the thoracic area, deep seated with no specific aggravating and relieving factor. The pain disturbed his sleep at night and affected his activities of daily living. The weakness of the both lower limb worsened as he became house-bound and it was associated with numbness of the limb. He had neither tuberculosis infection nor contact with tuberculosis patients. He had neither abdominal swelling nor difficulty in micturation. There was no family history of carcinoma of the lung and he was not a smoker.

Clinically, he looked pale, not cachexic and neurological examination of lower limbs revealed absent of the motor power, brisk reflexes bilaterally with reduced sensation from T7 level downward. Other systems examinations were normal. His hemoglobin was $7 \mathrm{~g} / \mathrm{dl}$ and the white cell was normal. Erythrocyte sedimentation 
rate (ESR) was $103 \mathrm{~mm} /$ hour and the Mantoux test was reactive. Radiograph of the thoracolumbar spine showed a collapse vertebra body of T7. Magnetic Resonance Imaging (MRI) of the thoracolumbar spine (Figure 1) was suggestive of an infective process of T6 to T8 with large paravertebral mass. He was treated as spinal tuberculosis with empirical anti-tuberculosis drugs. However, he did not respond to the chemotherapy and a computerized tomography (CT) guided biopsy of the paravertebral mass was performed. CTscans of the thorax revealed peripherally located speculated right lower lobe lesion consistent with lung carcinoma (Figure 2). Histopathology examination of the mass was consistent with adenocarcinoma of the lung (Figure 3).
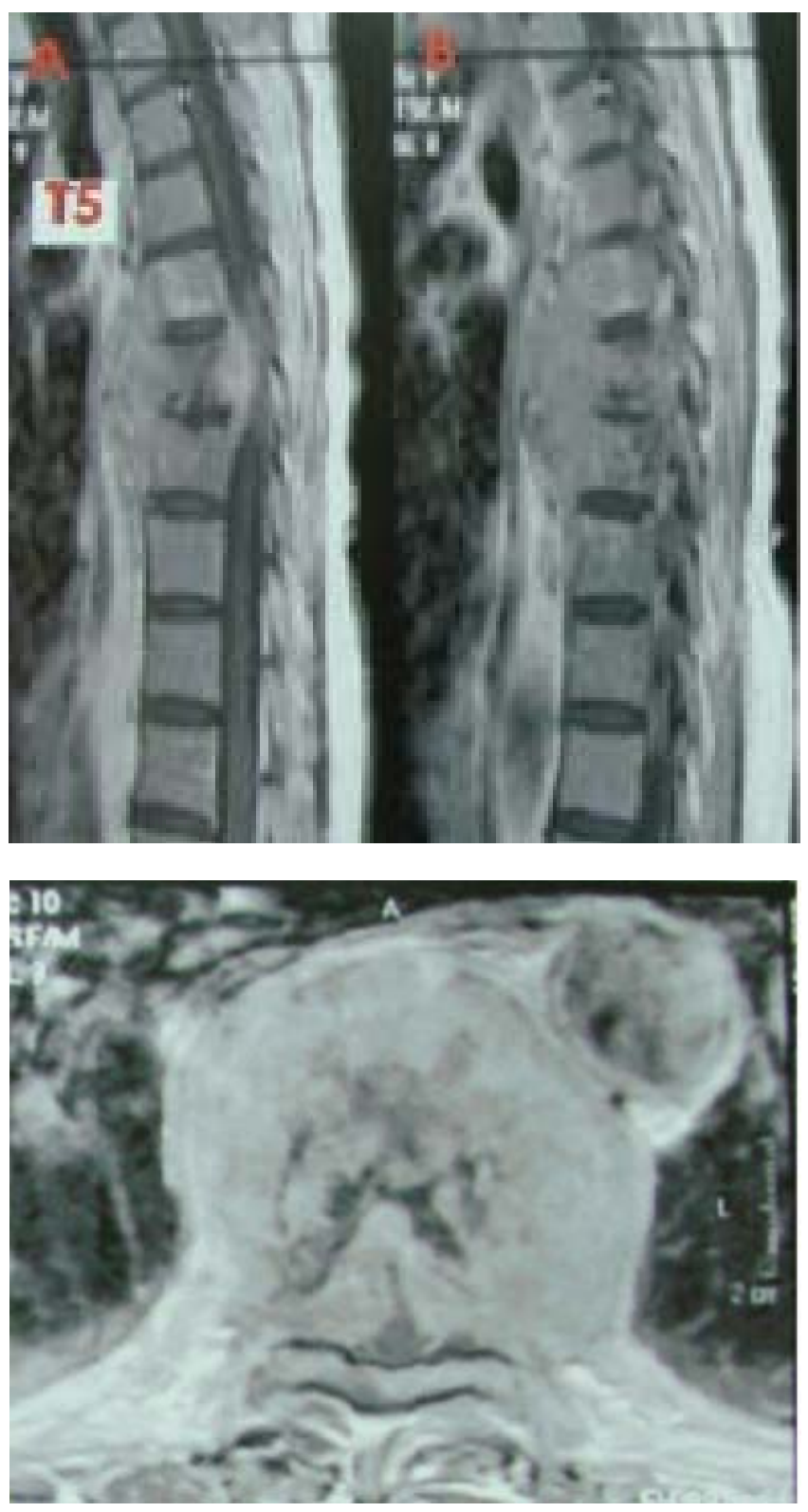

Figure 1.(A) Midsagittal T1 - weighted MRI before and (B) after gadolinium showed inhomogenous enhancement of T6 to T8 with destruction of T7 and paravertebral mass. On axial view, there is destruction of T7 with enhancing paravertebral mass. The spinal cord is severely compressed.

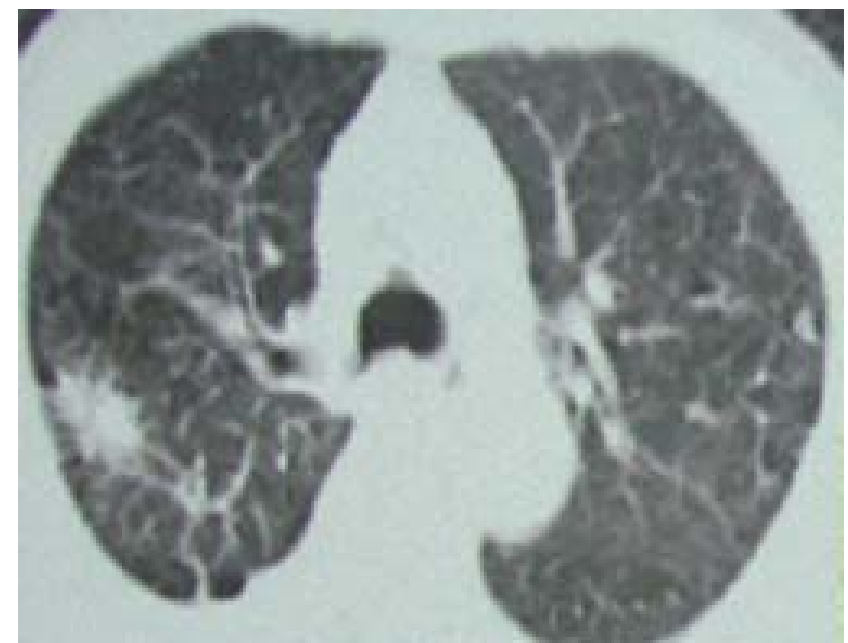

Figure 2. Axial post-contrast CT of thorax shows peripherally located specullated right lower lobe lesion consistent with lung car $\neg$ cinoma.

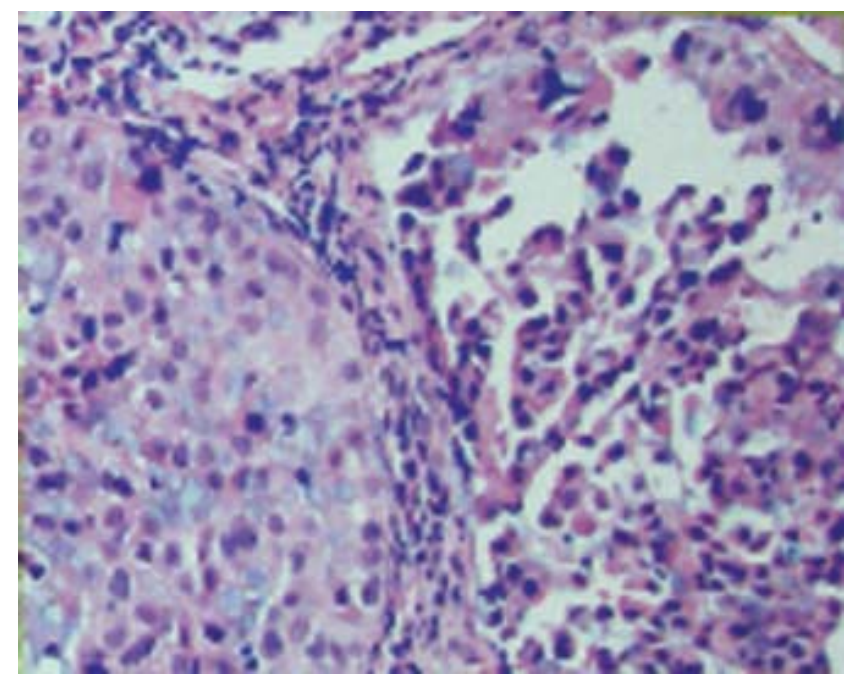

Figure 3. HPE of the paravertebral mass shows malignant tumour arranged as solid nest with glandular formation. The tumour cells show pleomorphic and hyperchromatic nuclei with moderate amount of cytoplasm. Mucin secretion is present in the tumour cells. (x20mag)

He was planned for decompression laminectomy and radiotherapy but he refused and opted for traditional medications.

\section{DISCUSSION}

The most common primary tumour that metastasis to spine is from the lung ranging from 14-31\%. Thoracic is the commonest location for metastases compare to the other vertebra and it accounts up to $70 \% .^{1}$ In this case, the patient presented with low back pain mainly at the thoracic vertebra, but he doesn't have neither any respiratory symptoms nor a risk factor such as chronic smoking habit or family history of malignancy to raise suspicions towards lung carcinoma. The value of erythrocyte sedimentation rate (ESR) of more than $100 \mathrm{~mm} / \mathrm{hr}$ will give an account towards malignancy or infection but the test is not specific. However, the reactive mantoux test doesn't really guide any 
clinicians towards a diagnosis of lung metastasis. The value of tuberculin skin test in the diagnosis of active tuberculosis is uncertain. It is still a sensitive but a non- specific test in the diagnosis of active tuberculosis. ${ }^{2}$ The clinical presentation of metastasis of the spine can mimic a presentation of an infection.

Magnetic resonance imaging (MRI) is very sensitive to pick up infection or metastasis. MRI findings in tuberculous spondylitis is commonly described as destruction of contiguous vertebral bodies, disc spaces involvement, end plates destruction and paravertebral abscesses. ${ }^{3}$ The other researchers reported characteristic MRI findings, including loss of cortical definition, intra-osseous abscesses with rim enhancement, paraspinal abscess, subligamentous spreading of inflammatory tissue with relative disc preservation. ${ }^{4,5}$ However, these findings are non-specific since in our case of spinal metastasis also gives the MRI appearances of an infection. This case illustrates that one must correlate with the clinical findings and do further investigations to confirm which entity is present: infection or metastasis.

The most reliable test for diagnosis is CT guided biopsy. It is a minimally invasive way to provide tissue for further analysis. Biopsy is recommended for patients who have spine lesions with no known primary malignant tumor or suspected spine infection without known organism. ${ }^{6}$ Presentation with spinal metastases may not be a terminal event. The aim of surgery is for pain relief and preservation or improvement of neurological functions. Surgical decompression with laminectomy is required when the spinal canal is compromised by vertebral collapse or infiltration of tumor and when there is spinal instability due to the pathological vertebral collapse or extensive destruction of the posterior elements, surgical stabilization with instrumentations may be needed. ${ }^{7}$

Radiotherapy is a key component of symptomatic treatment for pain from bone metastasis and advanced stage IV adenocarcinoma of lung. Although adenocarcinoma is much less chemosensitive than small cell lung carcinoma, chemotherapy can improve survival and quality of life in patients with adenocarcinoma if given in combination of two to three drugs. ${ }^{8,9}$

\section{CONCLUSION}

Metastatic lesion in the spine can mimic tuberculosis in clinical and imaging presentation. Biopsy is mandatory when in doubt of a diagnosis, especially in a case with atypical presentation or not responding to treatment before commencing with any form of treatment.

\section{REFERENCES}

1. Ufuk A, Cagatay O ,Sami B, et al. Evaluation of lung cancer metastasis to the spine. Acta Orthop Belg 2006; 72: 592-597.

2. Yaacob I, Ahmad Z. Clinical significance of Mantoux test in Malaysia patients. Med $\mathrm{J}$ Malaysia 1990; 45(3): 231-4.

3. Sharif HS, Clark DC, Albed MY, et al. Granulomatous spinal infections: MR Imaging. Radiology 1990; 177: 101-7.

4. Jung NY, Jee WH, Ha KY, et al. Discrimination of tuberculous spondylitis from pyogenic spondylitis on MRI. Am J Radiol 2004; 182: 1405-10.

5. Roos AD, Meeten EPV, Bloem JL, et al. MRI of tuberculous spondylitis. Am J Radiol 1986; 146: 79-82.

6. Eismont FJ, Green BA, Brown MD, et al. Coexistent infection and tumor of the spine. A report of three cases. J Bone Joint Surg Am 1987; 69: 452-458.

7. Bach F, Agerlin N, Sorensen JB, et al. Metastatic spinal cord compression secondary to lung cancer. J Clin Oncol 1992;10: 1781-1788.

8. Klimo PJ, Schmidt MH. Surgical management of spinalmetastases. Oncolo 2004;9(2):188196.

9. Parlids $\mathrm{N}$ et al. Diagnostic and therapeutic management of cancer of unknown primary. Eur J Cancer 2003; 39: 1990-2005. 
